\title{
Direct imaging of propagation and damping of near-resonance surface plasmon polaritons using cathodoluminescence spectroscopy
}

\author{
J. T. van Wijngaarden, E. Verhagen, and A. Polman ${ }^{a)}$ \\ Center for Nanophotonics, FOM Institute for Atomic and Molecular Physics, Kruislaan 407, \\ 1098 SJ Amsterdam, The Netherlands \\ C. E. Ross, H. J. Lezec, ${ }^{\text {b) }}$ and H. A. Atwater \\ Thomas J. Watson Laboratories of Applied Physics, California Institute of Technology, Pasadena, \\ California 91125
}

(Received 27 January 2006; accepted 19 April 2006; published online 1 June 2006)

\begin{abstract}
Cathodoluminescence imaging spectroscopy is used to determine the propagation distance of surface plasmon polaritons near the surface plasmon resonance on both silver and gold films. Surface plasmon polaritons are generated by a focused (diameter of $5 \mathrm{~nm}$ ) electron beam spot in the metal and coupled out through a grating. By gradually varying the distance between the excitation spot and the grating the damping is probed. Propagation lengths as small as several hundred nanometers are probed, and an increase in propagation length is observed if the wavelength is increased above resonance. The measured data are compared with the calculated propagation lengths taking into account both absorption in the film and leakage radiation, and it is found that other loss mechanisms appear to be significant as well. (C) 2006 American Institute of Physics.
\end{abstract}

[DOI: 10.1063/1.2208556]

Surface plasmon polaritons (SPPs) are electromagnetic waves that propagate at a metal-dielectric interface. ${ }^{1}$ Due to the strong dispersion of the metal's dielectric constant, the propagation length of SPPs is strongly frequency dependent. ${ }^{2}$ In the visible and (near) infrared, propagation distances as long as $10-100 \mu \mathrm{m}$ have been measured for silver and gold films. ${ }^{3}$ Near the surface plasmon resonance frequency, typically in the UV/visible, the propagation lengths are much smaller, and it becomes increasingly difficult to excite and measure the propagation of SPPs with conventional optical techniques. On the other hand, it is this region just below the surface plasmon resonance frequency that holds great potential for applications in photonics, because the wavelength of the SPPs in this region is much smaller than that of its photonic counterpart, the SPP group velocity is reduced, and the SPP field is highly concentrated. The near-resonance SPPs therefore have the potential to enable the scaling down of optoelectronic devices, to control the propagation of optical pulses, and to concentrate light. In view of these applications it is important to have knowledge of the propagation length of near-resonance SPPs.

In this letter we will show that a combination of scanning electron microscopy (SEM) and cathodoluminescence (CL) imaging spectroscopy can be used to generate SPPs on a nanometer scale and to measure the propagation distance of SPPs on thin metal films at length scales as small as several hundred nanometers. We excite SPPs by electron irradiation of a metal film ${ }^{4,5}$ at a well known distance from a nanoscale grating, that is made in the metal. The grating couples the SPP to the free space photon modes detected in the far field, and by varying the distance between excitation spot and grating the SPP damping can be probed [see schematic in Figs. 1(a) and 1(b)]. Experiments were performed on both silver

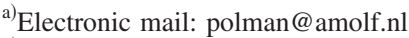

b) Also at: Centre National de la Recherche Scientifique, 3 rue Michel-Ange, 75796 Paris cedex 16, France.
}

and gold films, at frequencies ranging from close to resonance to the near infrared. The experimentally found propagation lengths were compared with the values expected on perfectly smooth films, calculated using the optical constants measured on the samples under study.

Thin silver and gold films patterned with gratings were fabricated in two different ways. A $400 \mathrm{~nm}$ thick silver layer was evaporated on a quartz substrate. With a focused ion beam (FIB) using a liquid Ga ion source, gratings with $500 \mathrm{~nm}$ pitch and a depth of $50 \mathrm{~nm}$ were then milled in the silver [Fig. 1(a)]. A second sample type was fabricated using electron beam lithography (EBL) to pattern grating structures with a pitch of $500 \mathrm{~nm}$ in a polymethyl methacrylate (PMMA) layer, spun on a silicon wafer. By developing the exposed PMMA and etching the silicon in a $\mathrm{SF}_{6}$ plasma, grating structures with an approximate depth of $70 \mathrm{~nm}$ were transferred to the silicon substrate. A gold film with a thick-
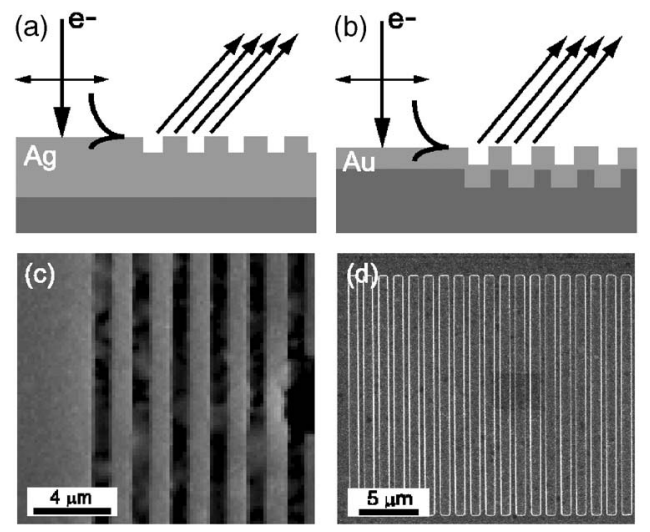

FIG. 1. Schematic of excitation and outcoupling of surface plasmon polaritons on samples made by (a) FIB milling (silver) and (b) e-beam writing (gold). The electron beam is incident normal to the metal film's surface and its distance from the grating is varied. Far-field radiation from the grating is then collected and analyzed. SEM images of the gratings in the silver and gold samples are shown in (c) and (d), respectively. 
ness of $70 \mathrm{~nm}$ was then deposited on the silicon by thermal evaporation [Fig. 1(b)]. Figures 1(c) and 1(d) show top view SEM images of gratings fabricated with FIB milling and EBL, respectively.

A $30 \mathrm{keV}$ electron beam from a FEI XL-30 SFEG SEM, with a beam diameter of approximately $5 \mathrm{~nm}$, was used to excite SPPs in the metal, close to the grating. The outcoupled light was collected by a parabolic mirror that was placed over the sample, that collects light in the angular range of $0^{\circ}-80^{\circ}$ from the surface normal. The electron beam was incident through a small opening in the mirror. An aluminum waveguide, attached to the mirror, led the light through the monochromator, that was connected to a photomultiplier tube.

When focusing the electron beam near a grating a broad spectrum was observed with a maximum at the surface plasmon resonance wavelength. The monochromator was set to several wavelengths above this resonance to compare the behavior of the CL intensity with varying beam position for different photon energies. CL intensity line profiles were either collected by the analysis of two-dimensional images, or using a single line scan.

Figure 2(a) shows the CL intensity collected from the silver film as a function of distance between the exciting electron beam and the edge of the grating. Data were collected at wavelengths of $\lambda=400,500$, and $600 \mathrm{~nm}$. A clear decay in intensity is observed as the electron beam moves away from the grating, and this intensity decay is slower for larger wavelengths. CL intensity profiles were also collected on gold films, and are plotted in Fig. 2(b) for $\lambda=550$ and $600 \mathrm{~nm}$. Signal-to-noise ratios proved to be smaller due to lower signal levels in this case, but similar intensity decays can be discerned at larger wavelengths than for silver.

We note that all CL data curves exhibited a significant background intensity that is constant far away from the grating. This may be attributed to radiation from SPPs that couple out from random surface roughness. In order to compare the CL intensity decays for different wavelengths, normalized intensity is plotted in Fig. 2 after the subtraction of these background values, determined using the fit procedure described below, and the curves are each shifted vertically for clarity.

To relate these CL intensity decays to the exponential decay of SPPs, we note that the electron beam generates SPPs in a point source; the SPPs subsequently propagate over a large angular range towards the grating [see inset in Fig. 2(b)]. The measured CL intensity as a function of distance between the excitation spot and the grating edge $x$ is therefore described by

$$
I(x)=I_{b}+I_{0} \int_{-\pi / 2}^{\pi / 2} \alpha(\theta) e^{-x / L_{\mathrm{SPP}} \cos \theta} d \theta,
$$

where $I_{b}$ is the background intensity, $I_{0}$ is the SPP intensity scattered at the grating edge, $L_{\mathrm{SPP}}$ is the SPP propagation length, and $\alpha(\theta)$ is the angle-dependent grating coupling efficiency. The latter is unknown, but assuming that it is a monotonously decreasing function of $\theta$, we can obtain upper and lower estimated limits to $L_{\mathrm{SPP}}$ from the measured $\mathrm{CL}$ intensity profiles using $\alpha(\theta)=1 / \pi$ and $\alpha(\theta)=\delta(\theta)$, respectively, where $\delta$ denotes the Dirac delta function. Both models were fitted to the CL data using nonlinear regression, yielding estimations of $L_{\mathrm{SPP}}$ and the background intensity $I_{b}$. The Downloaded 26 Jun 2006 to 192.87 .154 .163 . Redistribution subject
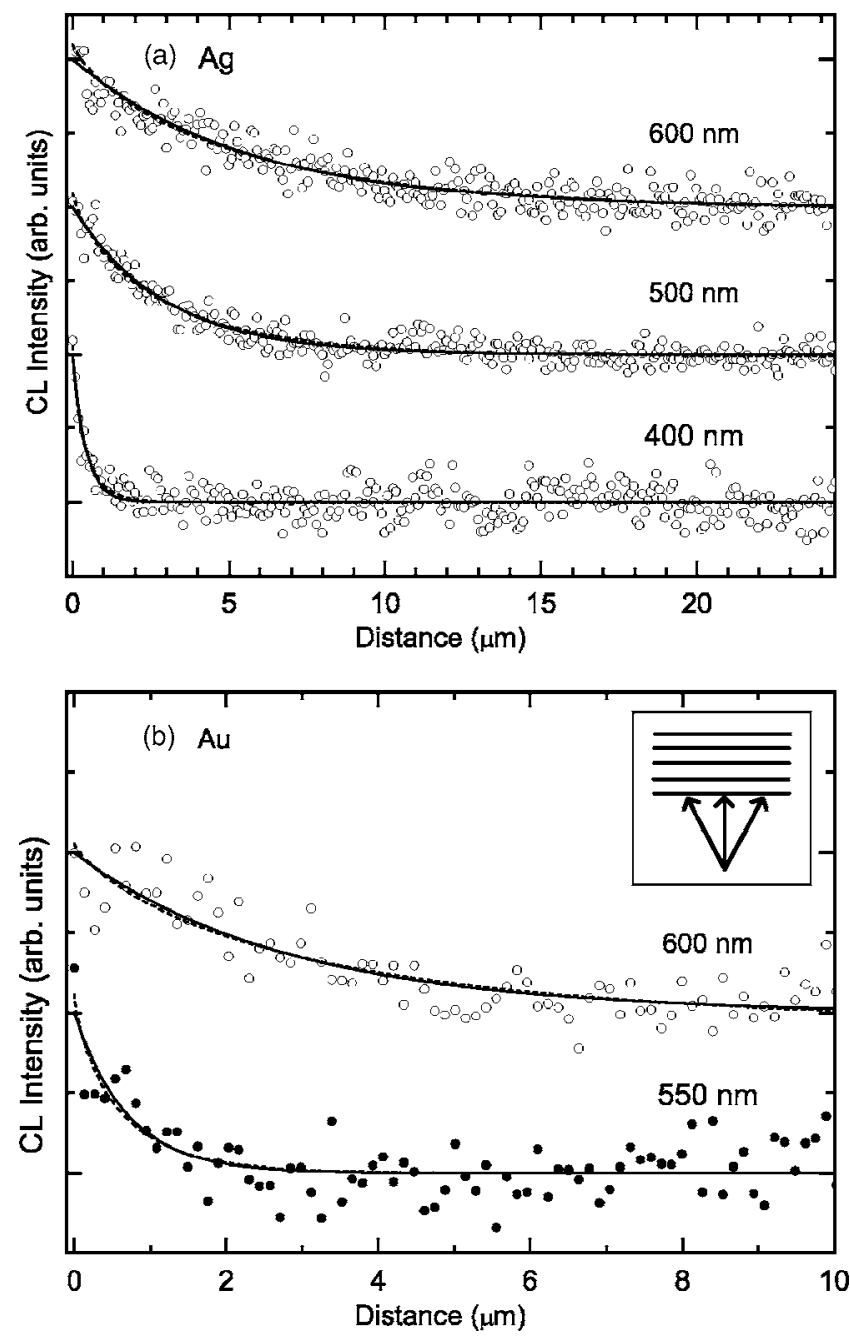

FIG. 2. Cathodoluminescence intensity (open and closed circles) as a function of distance between electron beam and grating edge for (a) a silver film, with profiles collected at $\lambda=400,500$, and $600 \mathrm{~nm}$, and (b) a gold film, with profiles collected at $\lambda=550$ and $600 \mathrm{~nm}$. A constant background is subtracted from all curves, that are then normalized and shifted vertically for clarity. Fits of Eq. (1) to the data using the angle-dependent grating coupling efficiencies $\alpha(\theta)=\delta(\theta)$ and $\alpha(\theta)=1 / \pi$ are shown as solid and dashed curves, respectively. Both models overlap in these plots in most cases. The inset in (b) schematically shows the SPPs propagating towards the grating over an angular range.

latter amounted to typically one to three times $I_{0}$. The fits to the data displayed in Fig. 2 are plotted through the data as solid and dashed curves for $\alpha(\theta)=\delta(\theta)$ and $\alpha(\theta)=1 / \pi$, respectively.

Measurements were also performed at other wavelengths, and the fitted upper and lower bounds to $L_{\mathrm{SPP}}$ are shown versus collection wavelength in Fig. 3. For both silver (closed symbols) and gold (open symbols), a clear increasing trend is observed as the wavelength moves away from resonance. Figure 3 also shows the calculated propagation lengths as a function of wavelength on perfectly smooth films. For the optically thick Ag film, this value is obtained by considering only bulk absorption losses: ${ }^{1}$

$$
L_{\mathrm{SPP}}=\left(2 k_{x}^{\prime \prime}\right)^{-1}=\frac{c}{2 \omega}\left[\operatorname{Im}\left(\sqrt{\frac{\varepsilon_{m}}{1+\varepsilon_{m}}}\right)\right]^{-1},
$$

with $k_{\mu}^{x}$ the imaginary part of the SPP wave vector and $\varepsilon_{m}$ the complex dielectric function of the metal.

to AIP license or copyright, see http://apl.aip.org/apl/copyright.jsp 


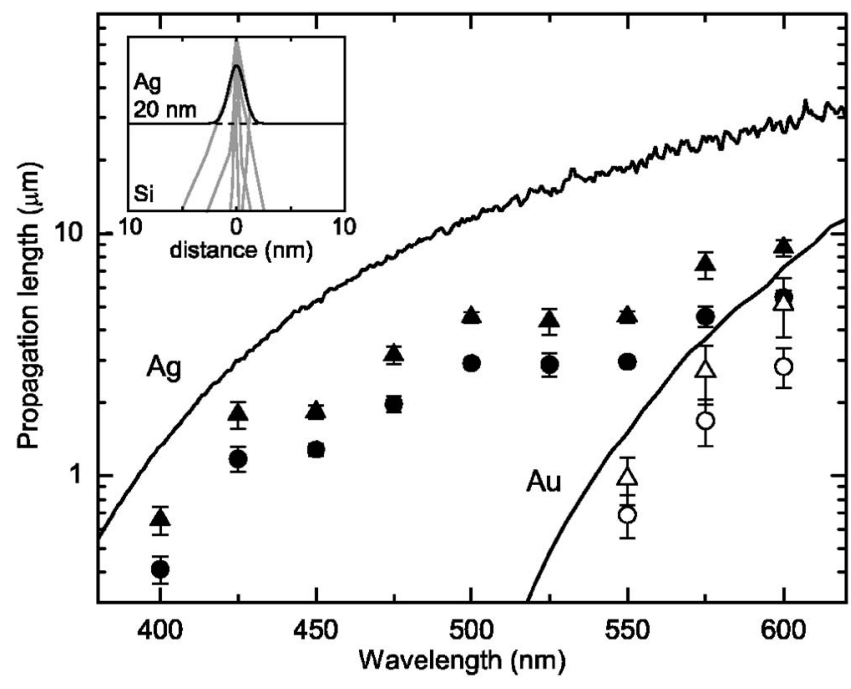

FIG. 3. SPP propagation length $L_{\mathrm{SPP}}$ fitted from CL intensity data as in Fig. 2 vs wavelength for silver (closed symbols) and gold (open symbols). The values obtained using $\alpha(\theta)=1 / \pi$ are represented by triangles, and those using $\alpha(\theta)=\delta(\theta)$ by circles. The drawn lines are the calculation of SPP propagation lengths for silver and gold using experimental data for the dielectric constant considering bulk absorption in the film and leakage radiation. The inset shows several $30 \mathrm{keV}$ electron trajectories in a $20 \mathrm{~nm}$ thick $\mathrm{Ag}$ film on $\mathrm{Si}$, and the radial distribution of these trajectories in a plane at a depth of $20 \mathrm{~nm}$.

For the $70 \mathrm{~nm}$ thick Au film, in addition to absorption losses, the SPPs propagating at the vacuum/metal interface lose energy to leakage radiation into the silicon substrate. The propagation length including this radiation loss was obtained from the imaginary part of the SPP wave vector calculated by solving the dispersion relation for the vacuum/ gold/silicon system. ${ }^{6}$ For both samples, the propagation lengths are calculated using experimental values for $\varepsilon_{m}$ determined from ellipsometry measurements on the metal films used. The surface plasmon resonance wavelengths predicted from these complex dielectric constants are $340 \mathrm{~nm}$ for silver and $510 \mathrm{~nm}$ for gold.

Irrespective of the functional form of $\alpha(\theta)$, a difference between observed and calculated dampings is observed, that can have its origin in several additional loss processes. One of them is surface roughness of the films, ${ }^{7}$ which causes in-plane scattering of SPPs and coupling of SPPs to far-field radiation. ${ }^{8}$ Additionally, grain boundaries and surface impurities could have a deteriorating effect on SPP propagation. The fact that the observed propagation lengths on the $\mathrm{Ag}$ sample appear to deviate further from the calculated values than those on the Au sample could arise from differences in surface morphologies and impurities. We refer a more quantitative analysis of these loss mechanisms to future studies.

These results show that CL imaging spectroscopy is a useful tool for high-resolution characterization of plasmonic nanostructures. It can be applied to study the propagation and damping in a whole array of SPP nanostructures, including SPP waveguides, tapers, and cavities. The ultimate resolution of this CL imaging technique is determined by the excitation volume in the metal film: thinner metal films will yield higher resolution. As an example, the inset in Fig. 3 shows a few trajectories of $30 \mathrm{keV}$ incident electrons on a $20 \mathrm{~nm}$ thick Ag film on Si calculated using a Monte Carlo simulation program ${ }^{9}$ and the distribution of these trajectories at a depth of $20 \mathrm{~nm}$. It shows that the spread of the electron beam in a $20 \mathrm{~nm}$ thick film is only a few nanometers, and thus that in such a geometry the lateral resolution is mostly determined by the incident beam diameter. More detailed calculations of the generation of SPP surface waves by the electron cascade inside the film are required to study the ultimate resolution that can be achieved. The smallest propagation length that can be probed using this technique is determined by the homogeneity at which the metal/grating interface can be fabricated.

In conclusion, we have determined the propagation length of surface plasmons near the surface plasmon resonance for both $\mathrm{Ag}$ and $\mathrm{Au}$ films. Propagation lengths as small as several hundred nanometers are determined. The propagation length increases as the wavelength is increased above resonance. Comparing the results with calculations taking into account loss due to absorption in the film and to leakage radiation shows that other loss mechanisms appear to have a large contribution as well. The ultimate imaging resolution of this technique will be determined by the excitation volume of SPPs by electrons, which may enable the characterization of SPPs and localized modes in a large array of plasmonic nanostructures.

Chris Retif is gratefully acknowledged for assistance in nanofabrication of the samples and Hans Zeijlemaker for the technical assistance during the installation of the CL system. This work was made possible by the fabrication and characterization facilities of the Amsterdam nanoCenter. It is part of the research program of the "Stichting voor Fundamenteel Onderzoek der Materie (FOM)," which is financially supported by the "Nederlandse Organisatie voor Wetenschappelijk Onderzoek (NWO)." It is also part of NANONED, a nanotechnology program of the Dutch Ministry of Economic Affairs. The Caltech portion of this work was supported by the Air Force Office of Scientific Research under MURI Grant No. FA9550-04-1-0434. One of the authors (C.R.) acknowledges fellowship support from the Department of Defense fellowship program, and another author (H.A.A.) acknowledges support from AMOLF's Joop Los Fellowship.

${ }^{1}$ H. Raether, Surface Plasmons On Smooth and Rough Surfaces and On Gratings (Springer, Berlin, 1988).

${ }^{2}$ J. A. Dionne, L. A. Sweatlock, H. A. Atwater, and A. Polman, Phys. Rev. B 72, 075405 (2005).

${ }^{3}$ B. Lamprecht, J. R. Krenn, G. Schider, H. Ditlbacher, M. Salerno, N. Felidj, A. Leitner, F. R. Aussenegg, and J. C. Weeber, Appl. Phys. Lett. 79, 51 (2001).

${ }^{4}$ N. Yamamoto, K. Araya, A. Toda, and H. Sugiyama, Surf. Interface Anal. 31, 79 (2001).

${ }^{5}$ D. Heitman, J. Phys. C 10, 397 (1977).

${ }^{6}$ J. J. Burke, G. I. Stegeman, and T. Tamir, Phys. Rev. B 33, 5186 (1986).

${ }^{7}$ A. Hoffmann, Z. Lenkefi, and A. Szentirmay, J. Phys.: Condens. Matter 10, 5503 (1998).

${ }^{8}$ D. L. Mills, Phys. Rev. B 12, 4036 (1975).

${ }^{9}$ P. Hovington, D. Drouin, and R. Gauvin, Scanning 19, 1 (1997). 